Author affiliations and support information (if applicable) appear at the end of this article.

Published at jco.org on March 14, 2017. Clinical trial information: NCT01631552.

Corresponding author: Aditya Bardia, MD MPH, Massachusetts General Hospital Cancer Center, Harvard Medical School, Lawrence House 304, 10 N Grove St, Boston, MA 02114; e-mail: bardia.aditya@ mgh.harvard.edu.

(C) 2017 by American Society of Clinical Oncology

$0732-183 X / 17 / 3519 w-2141 w / \$ 20.00$

\title{
Efficacy and Safety of Anti-Trop-2 Antibody Drug Conjugate Sacituzumab Govitecan (IMMU-132) in Heavily Pretreated Patients With Metastatic Triple-Negative Breast Cancer
}

Aditya Bardia, Ingrid A. Mayer, Jennifer R. Diamond, Rebecca L. Moroose, Steven J. Isakoff, Alexander N. Starodub, Nikita C. Shah, Joyce O’Shaughnessy, Kevin Kalinsky, Michael Guarino, Vandana Abramson, Dejan Juric, Sara M. Tolaney, Jordan Berlin, Wells A. Messersmith, Allyson J. Ocean, William A. Wegener, Pius Maliakal, Robert M. Sharkey, Serengulam V. Govindan, David M. Goldenberg, and Linda T. Vahdat

\section{$\begin{array}{llllllll}\text { A } & \text { B } & \mathbf{S} & \mathbf{T} & \mathbf{R} & \mathbf{A} & \mathbf{C} & \mathbf{T}\end{array}$}

\section{Purpose}

Trop-2, expressed in most triple-negative breast cancers (TNBCs), may be a potential target for antibody-drug conjugates. Sacituzumab govitecan, an antibody-drug conjugate, targets Trop-2 for the selective delivery of SN-38, the active metabolite of irinotecan.

\section{Patients and Methods}

We evaluated sacituzumab govitecan in a single-arm, multicenter trial in patients with relapsed/ refractory metastatic TNBC who received a $10 \mathrm{mg} / \mathrm{kg}$ starting dose on days 1 and 8 of 21-day repeated cycles. The primary end points were safety and objective response rate; secondary end points were progression-free survival and overall survival.

\section{Results}

In 69 patients who received a median of five prior therapies (range, one to 12) since diagnosis, the confirmed objective response rate was $30 \%$ (partial response, $n=19$; complete response, $n=2$ ), the median response duration was $8.9(95 \% \mathrm{Cl}, 6.1$ to 11.3$)$ months, and the clinical benefit rate (complete response + partial response + stable disease $\geq 6$ months) was $46 \%$. These responses occurred early, with a median onset of 1.9 months. Median progression-free survival was $6.0(95 \%$ $\mathrm{Cl}, 5.0$ to 7.3) months, and median overall survival was 16.6 (95\% Cl, 11.1 to 20.6) months. Grade $\geq 3$ adverse events included neutropenia (39\%), leukopenia (16\%), anemia (14\%), and diarrhea (13\%); the incidence of febrile neutropenia was $7 \%$. The majority of archival tumor specimens (88\%) were moderately to strongly positive for Trop-2 by immunohistochemistry. No neutralizing antibodies to the ADC or antibody were detected, despite repeated cycles developed.

\section{Conclusion}

Sacituzumab govitecan was well tolerated and induced early and durable responses in heavily pretreated patients with metastatic TNBC. As a therapeutic target and predictive biomarker, Trop-2 warrants further research.

\section{J Clin Oncol 35:2141-2148. (C) 2017 by American Society of Clinical Oncology}

\section{INTRODUCTION}

Despite considerable progress in management over the past few decades, ${ }^{1}$ breast cancer still carries a high mortality that accounted for 521,900 deaths worldwide in 2012. ${ }^{2}$ Triplenegative breast cancer (TNBC), defined as the absence of estrogen and progesterone receptors and lack of human epidermal growth factor receptor 2 (HER2) gene amplification, comprises $15 \%$ to $20 \%$ of breast cancers. ${ }^{3,4}$ TNBC has a high mortality rate because of its aggressive behavior, with a median survival of 10 to 13 months from time of metastasis. ${ }^{4-7}$

TNBC disproportionally affects younger patients, particularly premenopausal African American women, and frequently displays homologous recombination deficiency and high genomic instability ${ }^{5,6}$ that may predict sensitivity to DNA-damaging agents, such as platinum and DNA repair inhibitors, including poly(ADPribose) polymerase-1 inhibitors. ${ }^{5,7,8}$ The management of TNBC could be improved by the development of specific targeted agents because most available drugs achieve progression-free 
survival (PFS) of $<3.5$ months. ${ }^{4,5,8-15}$ Therefore, we evaluated an antibody-drug conjugate (ADC), sacituzumab govitecan (IMMU132), that targets Trop-2, a glycoprotein elevated in various solid cancers, including TNBC. ${ }^{16-20}$

Trop-2 is a $46-\mathrm{kD}$ glycoprotein initially identified in a trophoblast cancer cell line $\mathrm{e}^{21}$ and is overexpressed in many epithelial cancers. ${ }^{18-20,22}$ It plays a multifunctional cellular role, including the transducing of cytoplasmic $\mathrm{Ca}^{2+}$ that depends on a specific protein kinase $\mathrm{C}$ phosphorylation site. ${ }^{23}$ Both Trop-2 and the biscistronic CYCLIN D1-Trop-2 mRNA chimera have oncogenic properties. ${ }^{24,25}$ The overexpression of Trop- 2 correlates with a poor prognosis in several cancers, ${ }^{19,20}$ including breast cancer. ${ }^{20,25}$

Sacituzumab govitecan comprises a toxic payload, SN-38 (7-ethyl-10-hydroxycamptothecin), which is a topoisomerase I-inhibiting drug that causes double-stranded DNA breaks that lead to apoptosis. ${ }^{26}$ Irinotecan, the prodrug of SN-38, has activity in solid tumors, including metastatic breast cancer, ${ }^{27,28}$ but its complex pharmacology limits delivery of SN-38, which has a 100- to 1,000fold higher potency than irinotecan and contributes to poor tolerability, with approximately one third of patients experiencing grade 3 to 4 diarrhea. ${ }^{29}$ In contrast, sacituzumab govitecan can deliver higher levels of SN-38 to tumors with an improved therapeutic index. ${ }^{16-18,30}$ A phase I dose-finding trial in advanced solid cancers, including metastatic TNBC (mTNBC), showed encouraging therapeutic activity without preselection of patients on the basis of Trop-2 expression by their tumors. ${ }^{31}$ The phase II portion of the study expanded accrual in select cancers. This article presents results for all patients with mTNBC who received sacituzumab govitecan at the $10 \mathrm{mg} / \mathrm{kg}$ dose level selected for development.

\section{PATIENTS AND METHODS}

\section{Patients}

We enrolled female or male patients $\geq 18$ years of age who had mTNBC refractory to or relapsed after at least one standard line of therapy since diagnosis and measurable disease by computed tomography scan (or magnetic resonance imaging). TNBC status was confirmed locally according to American Society of Clinical Oncology/ American College of Pathology guidelines. ${ }^{31 a, 31 b}$

Eligible patients had an Eastern Cooperative Oncology Group performance status of 0 or 1 , adequate bone marrow, hepatic and renal function, and prior toxicities at study entry of grade $\leq 1$ by National Cancer Institute Common Terminology Criteria for Adverse Events (NCI-CTCAE) version 4.03. Patients with brain metastasis were excluded, unless treated and without progression, and were not receiving high-dose corticosteroids for at least 4 weeks; other enrollment criteria have been described previously. ${ }^{31}$

\section{Study Oversight}

The protocol was approved by the participating institutions' review boards and conducted in accordance with the Declaration of Helsinki and the International Conference on Harmonization Good Clinical Practice guidelines. All patients provided written informed consent. Immunomedics sponsored the trial, which was designed through a collaboration of the sponsor and the lead investigators. All authors vouch for the accuracy of the data and analysis and adherence of the trial to the protocol.

\section{Study Design and Treatment}

The study objectives were to evaluate safety and antitumor activity of sacituzumab in patients with advanced epithelial cancer, including mTNBC.
The protocol was a phase I and II basket design, single-arm, multicenter study that enrolled patients with many different cancer types (including patients with mTNBC), all of whom received sacituzumab govitecan administered intravenously on days 1 and 8 of 21-day cycles. The study population comprised all patients from the phase II portion and one patient given $10 \mathrm{mg} / \mathrm{kg}$ from the phase I trial. Objective response rate (ORR) was measured by Response Evaluation Criteria in Solid Tumors version 1.1 (RECIST 1.1). Treatment was continued as long as evidence of clinical benefit existed and discontinued as a result of progression, unacceptable toxicity, investigator or patient decision, or death. In patients without disease progression, a treatment break after eight cycles was permitted after discussion with the sponsor. Toxicities were managed in accordance with standard medical practice and/or by dose modification specified in the protocol.

\section{Study Assessments}

Safety was evaluated weekly according to NCI-CTCAE version 4.03 . Tumor response was assessed locally (including use of radiology core laboratories) every 8 weeks until disease progression, with a partial response $(\mathrm{PR})$ or complete response $(\mathrm{CR})$ requiring confirmation within 4 to 6 weeks after the initial response, as required by RECIST 1.1 for single-arm studies.

\section{Tumor Trop-2 Immunohistochemistry and Immunogenicity}

Available archival tumor specimens were stained for Trop-2 by immunohistochemistry (IHC) as reported previously. ${ }^{31}$ Positivity required at least $10 \%$ of the tumor cells to be stained, with an intensity scored of $1+$ (weak), $2+$ (moderate), and $3+$ (strong). Examples of cases with $1+, 2+$, and $3+$ staining for Trop-2 are shown in Appendix Figure A1 (online only). Antibody responses to sacituzumab govitecan, the antibody, and $\mathrm{SN}-38$ were monitored in serum samples taken at baseline and then before each even numbered cycle by enzyme-linked immunosorbent assays performed by the sponsor, as previously reported. ${ }^{31}$ Although the Trop-2 results were not required for eligibility, Trop-2 analysis on prior (archived) biopsy or surgical specimens was prespecified. The association between Trop-2 and clinical outcomes was done as post hoc and exploratory analyses.

\section{Statistics}

The study enrolled the number of patients that would provide $90 \%$ power to exclude an unacceptably low $10 \%$ ORR (the null hypothesis) with $95 \%$ confidence if the true rate is $25 \%$. On the basis of a Simon two-stage optimal design, 82 patients with TNBC who had received at least one standard line of therapy were initially planned to be enrolled. However, we observed a much-higher-than-anticipated efficacy. With 69 patients enrolled, the statistical design requirements for efficacy, on the basis of an independent statistical review, were met, and the null hypothesis was rejected with $>99.9 \%$ confidence.

All patients administered any dose of study drug were included in the safety evaluation. The frequency and severity of adverse events (AEs) were tabulated by Medical Dictionary for Regulatory Activities Preferred Term and System Organ Class for each dose group. AEs were classified using the Medical Dictionary for Regulatory Activities version $10^{31 \mathrm{c}}$, with severity assessed by NCI-CTCAE version 4 toxicity grades. AEs that led to death or to discontinuation from treatment also were summarized separately.

To be included in the assessment of treatment response, the protocol required completion of at least one treatment cycle, but we included all patients on an intention-to-treat basis per RECIST 1.1 recommendations. Response duration was defined from the time of the first evidence of response until progression, whereas PFS and OS were defined from time of the first dose of sacituzumab govitecan to progression or death (PFS) or death alone (OS). Duration of response, PFS, and OS were determined by Kaplan-Meier method by using MedCalc version 16.4.3 statistical software (MedCalc Software, Ostend, Belgium). 


\section{RESULTS}

\section{Patients}

Between July 2013 and March 2016, we enrolled 69 patients with mTNBC (68 females, one male). Demographics are listed in Table 1 . These patients had at least one prior therapy since diagnosis and were in the dosing cohort of $10 \mathrm{mg} / \mathrm{kg}$. The median age was 56 years (range, 31 to 81 years). Most patients had extensive metastatic disease and were heavily pretreated (median of five lines of therapy since diagnosis; range, one to 12), with the majority having received taxanes, anthracyclines, and platinum agents. Prior treatment with PD-1 (programmed cell death) or PD-L1 (programmed cell death ligand) immune checkpoint inhibitor antibodies also were given to four patients as the last therapy before enrollment.

\section{Treatment Administration and AEs}

At the data cutoff on August 2, 2016, with a median follow-up of 16.6 months, 62 patients had discontinued treatment, and seven

\begin{tabular}{|c|c|}
\hline Characteristic & $\begin{array}{l}\text { Patients, } \\
\text { No. (\%) }\end{array}$ \\
\hline \multicolumn{2}{|l|}{ Sex } \\
\hline Female & 68 (99) \\
\hline Male & $1(1)$ \\
\hline Median age, years (range) & $56(31-81)$ \\
\hline \multicolumn{2}{|l|}{ Ethnicity } \\
\hline White & $57(83)$ \\
\hline Black & $4(6)$ \\
\hline Asian & 2 (3) \\
\hline Other/not specified & $6(9)$ \\
\hline \multicolumn{2}{|l|}{ ECOG performance status } \\
\hline 0 & $23(33)$ \\
\hline 1 & $46(67)$ \\
\hline \multicolumn{2}{|l|}{ Stage at initial diagnosis* } \\
\hline 1 & $12(17)$ \\
\hline II & $25(36)$ \\
\hline III & $21(30)$ \\
\hline IV & $10(14)$ \\
\hline Median No. of prior therapies (range) & $5(1-12)$ \\
\hline \multicolumn{2}{|l|}{ Prior chemotherapy drugs (> $>$ 10\%) } \\
\hline Taxanes & $67(97)$ \\
\hline Cyclophosphamide & $63(91)$ \\
\hline Anthracyclines & $58(84)$ \\
\hline Platinum agents & $48(70)$ \\
\hline Fluoropyrimidine agents & $34(49)$ \\
\hline Gemcitabine & $30(43)$ \\
\hline Eribulin & 27 (39) \\
\hline Vinorelbine & $13(19)$ \\
\hline Ixabepilone & $8(12)$ \\
\hline \multicolumn{2}{|l|}{ Sites of disease ${ }^{-}$} \\
\hline Lymph node & $43(62)$ \\
\hline Lung & $35(51)$ \\
\hline Liver & $30(43)$ \\
\hline Chest & $28(41)$ \\
\hline Bone & $21(30)$ \\
\hline Skin & $7(10)$ \\
\hline \multicolumn{2}{|c|}{$\begin{array}{l}\text { Abbreviation: ECOG, Eastern Cooperative Oncology Group. } \\
\text { * Original stage not available for one patient with initial surgically resectable local } \\
\text { disease. } \\
\text { †As reported by baseline imaging Response Evaluation Criteria in Solid Tumors } \\
\text { (RECIST) version } 1.1 \text { assessment. }\end{array}$} \\
\hline
\end{tabular}

still received therapy. A median of 14 doses were administered per patient (range, one to 67), with a median duration of exposure of 5.3 months (range, 0.2 to 23.1 months). To prevent potential infusion reactions, most patients had coadministration of premedication that included an antihistamine; $56 \%$ also had a corticosteroid added.

Of the 69 patients, one or more grade $\geq 3 \mathrm{AEs}$ developed in $41 \%$ and were predominantly neutropenia $(39 \%)$, but the incidence of febrile neutropenia was $7 \%$ (Table 2). Other grade 3 to 4 AEs were leukopenia (16\%), anemia (14\%), diarrhea (13\%), and vomiting (10\%); grade 3 thrombocytopenia occurred in two patients (3\%). Three patients discontinued treatment because of AEs after six to seven doses (grade $3 \mathrm{rash} /$ mucositis, grade 3 transient infusion reaction, grade 2 fatigue after six doses). No therapyrelated deaths occurred.

Dose reductions, primarily as a result of neutropenia, occurred in 13 patients (19\%) in the first two cycles (either dose 2 or 3), with an additional $11(16 \%)$ requiring later dose reductions. No neutralizing antibodies to the ADC SN-38 or to the antibody were detected, including after the administration of multiple therapy cycles.

\section{Overall Efficacy}

Of the 69 patients, three withdrew as a result of early progression after receiving only one or two doses without a postbaseline computed tomography assessment, but they were included in the intention-totreat analysis. Overall, $30 \%$ (21 of 69) achieved confirmed objective responses (19 PRs, two CRs) with a $95 \%$ CI of $20 \%$ to $43 \%$ (Table 3), and $69.5 \%$ (48 of 69) experienced a reduction of tumor burden as shown in the waterfall plot in Fig 1A. The median time to an objective response was 1.9 months (range, 1.3 to 13.4 months), with 13 (62\%)

\begin{tabular}{|c|c|c|}
\hline Adverse Event & All Grades, No. (\%) & Grades $\geq 3$, No. (\%) \\
\hline Nausea & $51(74)$ & $5(7)$ \\
\hline Neutropenia & 47 (68) & 27 (39) \\
\hline Diarrhea & $41(59)$ & $9(13)$ \\
\hline Anemia & $38(55)$ & $10(14)$ \\
\hline Vomiting & $35(51)$ & $7(10)$ \\
\hline Fatigue & $35(51)$ & $6(9)$ \\
\hline Alopecia & $31(45)$ & $0(0)$ \\
\hline Constipation & $26(38)$ & $1(1)$ \\
\hline Rash & $19(28)$ & $1(1)$ \\
\hline Abdominal pain & $18(26)$ & $2(3)$ \\
\hline Leukopenia & $17(25)$ & $11(16)$ \\
\hline Dyspnea & $16(23)$ & $6(9)$ \\
\hline Back pain & $16(23)$ & $0(0)$ \\
\hline Anorexia & $16(23)$ & $0(0)$ \\
\hline Urinary tract infection & $16(23)$ & $2(3)$ \\
\hline Hypomagnesemia & $15(22)$ & $0(0)$ \\
\hline Dehydration & 13 (19) & $4(6)$ \\
\hline Dizziness & 13 (19) & $1(1)$ \\
\hline Headache & $13(19)$ & $1(1)$ \\
\hline Hyperglycemia & $13(19)$ & $1(1)$ \\
\hline Hypokalemia & 13 (19) & $1(1)$ \\
\hline Hypophosphatemia & $12(17)$ & $7(10)$ \\
\hline Elevated ALP & $11(16)$ & $2(3)$ \\
\hline Arthralgia & $11(16)$ & $0(0)$ \\
\hline Febrile neutropenia & $5(7)$ & $5(7)$ \\
\hline \multicolumn{3}{|c|}{$\begin{array}{l}\text { NOTE. Frequency of } 69 \text { patients with events regardless of causality that oc- } \\
\text { curred in }>15 \% \text { (all grades) or }>3 \% \text { (grades } \geq 3 \text { ) of patients. Abbreviation: ALP, } \\
\text { alkaline phosphatase. }\end{array}$} \\
\hline
\end{tabular}


Table 3. Treatment Efficacy in Intention-to-Treat Data Set $(N=69)$

\begin{tabular}{lc}
\hline \multicolumn{1}{c}{ Efficacy } & $\begin{array}{c}\text { Best Overall Response, } \\
\text { No. (\%) }\end{array}$ \\
\hline $\mathrm{CR}$ & $2(3)$ \\
$\mathrm{PR}$ & $19(28)$ \\
$\mathrm{SD}$ & $31(45)$ \\
$\mathrm{PD}$ & $17(25)$ \\
Confirmed objective response (CR + PR) & $21(30)$ \\
95\% Cl & 20 to 43 \\
Clinical benefit (CR + PR + SD $\geq 6$ months) & $32(46)$ \\
95\% Cl & 34 to 59 \\
Median duration of objective response, months & $8.9(6.1$ to 11.3$)$ \\
(95\% Cl) & $6.0(5.0$ to 7.3$)$ \\
Median PFS, months (95\% Cl) & $16.6(11.1$ to 20.6) \\
Median OS, months (95\% Cl)
\end{tabular}

Abbreviations: $C R$, complete response; OS, overall survival; $P D$, progressive disease; PFS, progression-free survival; PR, partial response; SD, stable disease.

\section{Trop-2 Staining}

Forty-eight patients had archival tumors (60\% primary tumors, $40 \%$ miscellaneous metastases) evaluated for Trop-2 expression. Of these, $42(88 \%)$ had moderate $(2+)$ to strong $(3+)$ Trop-2 staining, with the majority expressing Trop-2 in $>50 \%$ of tumor cells, whereas only six $(12 \%)$ had weak $(1+; n=4)$ or no Trop-2 staining $(n=2)$. Forty-six of these patients had a response assessment; the other two withdrew before having their first response assessment. All responders had moderate to strong Trop-2 staining, whereas patients whose specimens had only weak $(1+)$ or no Trop-2 staining had SD as the best response (Table 4). Among all 48 patients, the trend was toward a higher PFS in those with moderate to strong Trop-2 staining than in those with weak to no Trop-2 (median PFS, $7.1 v$ 3.1 months; $P=.019$; Appendix Fig A2, online only). However, the number of specimens with $1+$ or no staining was limited $(n=6)$, and results should be interpreted with caution.

\section{DISCUSSION}

of the 21 responders achieving $>30 \%$ reduction at the first response assessment at 8 weeks (Fig 1B). Nine (43\%) of the 21 confirmed responders continued treatment for at least 12 months, and the median duration of response was 8.9 months (95\% CI, 6.1 to 11.3 months; Table 3). Figure 1C illustrates the time course of tumor response, with the majority of patients having durable responses and three having as long as approximately 20 months. Because 11 patients had stable disease (SD) for at least 6 months, the clinical benefit rate ( $\mathrm{CR}+\mathrm{PR}+\mathrm{SD} \geq 6$ months) was $46 \%$ (Table 3 ).

Three of the four patients who had received prior immune checkpoint inhibitor therapy achieved a PR with sacituzumab govitecan (one confirmed). Only one of these patients had an antitumor response of 4 months duration to the anti-PD-L1 antibody.

The median PFS at the cutoff was 6.0 months (95\% CI, 5.0 to 7.3 months; Fig 1D), and the median OS was 16.6 months (95\% CI, 11.1 to 20.6 months; Fig 1E; Table 3 ). These findings include two patients whose disease progressed per RECIST 1.1 with new brain lesions but who continued sacituzumab govitecan therapy after whole-brain irradiation. One patient's non-CNS target lesions remained stable for an additional 9.1 months, and the other's nonCNS target lesions maintained a PR for 7.5 months.

Examples of treatment responses are shown in Fig 2. The first patient had four prior therapy regimens, including two in the metastatic setting, with a PD-L1 inhibitor being the last treatment. She achieved a PR within 1.7 months, with a maximum tumor reduction of 54\% (Figs 2A to 2C). The duration of response was 14.4 months (42 doses given). Another patient had a PR for 10.8 months, with a $66 \%$ reduction as the best response, including resolution of her extensive skin involvement and significant improvement in cancerrelated pain (Figs $2 \mathrm{D}$ to $2 \mathrm{~J}$ ). A third patient had six prior cytotoxic regimens and achieved an ongoing PR of 13.2 months duration.

\section{BRCA Status}

Of 43 patients with assessable BRCA1 germline status at enrollment, seven $(16 \%)$ had mutations with one PR, four SD, and two progressive disease, whereas $36(84 \%)$ had BRCA1 wild-type genes with nine $\mathrm{CR} / \mathrm{PR}, 15 \mathrm{SD}$, and 12 progressive disease, resulting in an ORR of $14 \%$ (one of seven) versus $25 \%$ (nine of 36), respectively.
We identified Trop-2 as a novel target in TNBC that can be exploited therapeutically with an ADC. Sacituzumab govitecan was well tolerated and led to early and durable objective responses.

The efficacy and safety results suggest that sacituzumab govitecan has meaningful clinical activity and a manageable safety profile and can be administered for long periods through 21-day cycles of dosing on days 1 and 8 without becoming immunogenic. We enrolled patients with extensive metastatic disease who were heavily pretreated (median of five prior regimens) with standard breast cancer drugs, including taxanes, anthracyclines, and platinum agents. In addition, four patients had previously received an immune checkpoint inhibitor, and although only one had responded briefly to this modality, three had a PR with sacituzumab govitecan. This anecdotal observation suggests that the ADC and PD-1/PD-L1 antibodies represent non-cross-resistant therapeutic options for a potential combination therapy, which requires further study.

Overall, approximately one third of the patients achieved a confirmed objective response, and one half had $a \geq 6$-month clinical benefit rate with sacituzumab govitecan therapy. The median PFS of 6 months compares favorably with the PFS of approximately 3.5 months reported in earlier treatment settings in mTNBC trials 16.6 months, despite being in a heavily pretreated population, compares favorably with a previously reported OS of $<13$ months after second-line therapy. ${ }^{4-7}$

ADCs represent an emerging therapeutic modality where antibodies are used as vehicles for carrying cytotoxic agents selectively to tumors, such as DM-1 linked with trastuzumab in trastuzumab emtansine. ${ }^{32-35}$ With sacituzumab govitecan, the cytotoxic drug is $\mathrm{SN}-38$, the active metabolite of irinotecan that is a topoisomerase I inhibitor with 2 to 3 logs higher potency than irinotecan. ${ }^{29}$ Although irinotecan has broad anticancer activity, ${ }^{26}$ it has had limited use as a monotherapy because of a modest therapeutic index (high rates of neutropenia and severe diarrhea). ${ }^{36}$ The humanized anti-Trop-2 hRS7 monoclonal antibody was derived from a murine $\operatorname{IgG}_{\mathrm{K} 1} \cdot{ }^{37} \mathrm{~A}$ concern of Trop-2 expression in normal tissues has been raised, ${ }^{38}$ but the that evaluated standard agents, including cisplatin, capecitabine, nab-paclitaxel, and eribulin. ${ }^{4,5-14}$ Similarly, the median OS of 


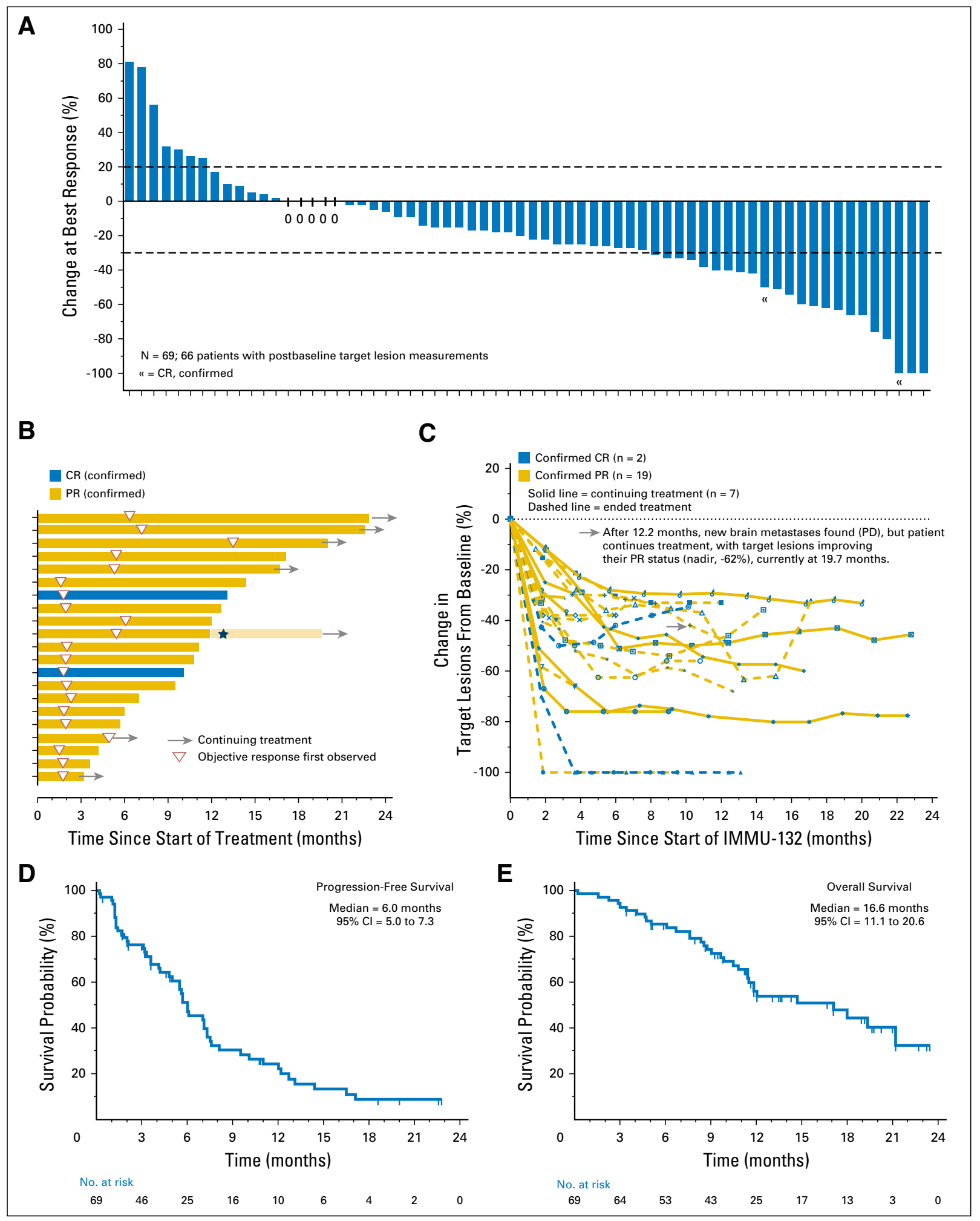

Fig 1. Efficacy assessments. (A) A waterfall plot that shows the percentage of change in sum of target lesions at the time of best response for 66 of the 69 patients who had at least one postbaseline computed tomography assessment. Forty-eight patients had reductions from baseline, including 23 with at least a $30 \%$ reduction (two confirmed complete responses [CRs], 19 confirmed partial responses [PRs], two unconfirmed PRs classified as stable disease by Response Evaluation Criteria in Solid Tumors [RECIST] version 1.1). (B) A swimmer graph that depicts the duration of treatment, onset, and duration of response for 21 responders. Fourteen patients progressed and discontinued treatment at that time (bars without arrows). Six have continuing response and treatment at this time (bars with arrows). One who progressed with new brain lesions after 12.2 months of treatment (star) but after localized radiation to the brain currently continues treatment cycles (bar with arrow) with additional shrinkage to body target lesions. (C) Percentage of change in sum of target lesions during treatment of each of the 21 responders; the gray arrow indicates the time course for the patient with brain metastases. (D) Kaplan-Meier progression-free survival graph for all 69 patients, with 63 having progressed. (E) Kaplan-Meier overall survival graph for all 69 patients, with 33 deceased. PD, progressive disease. 


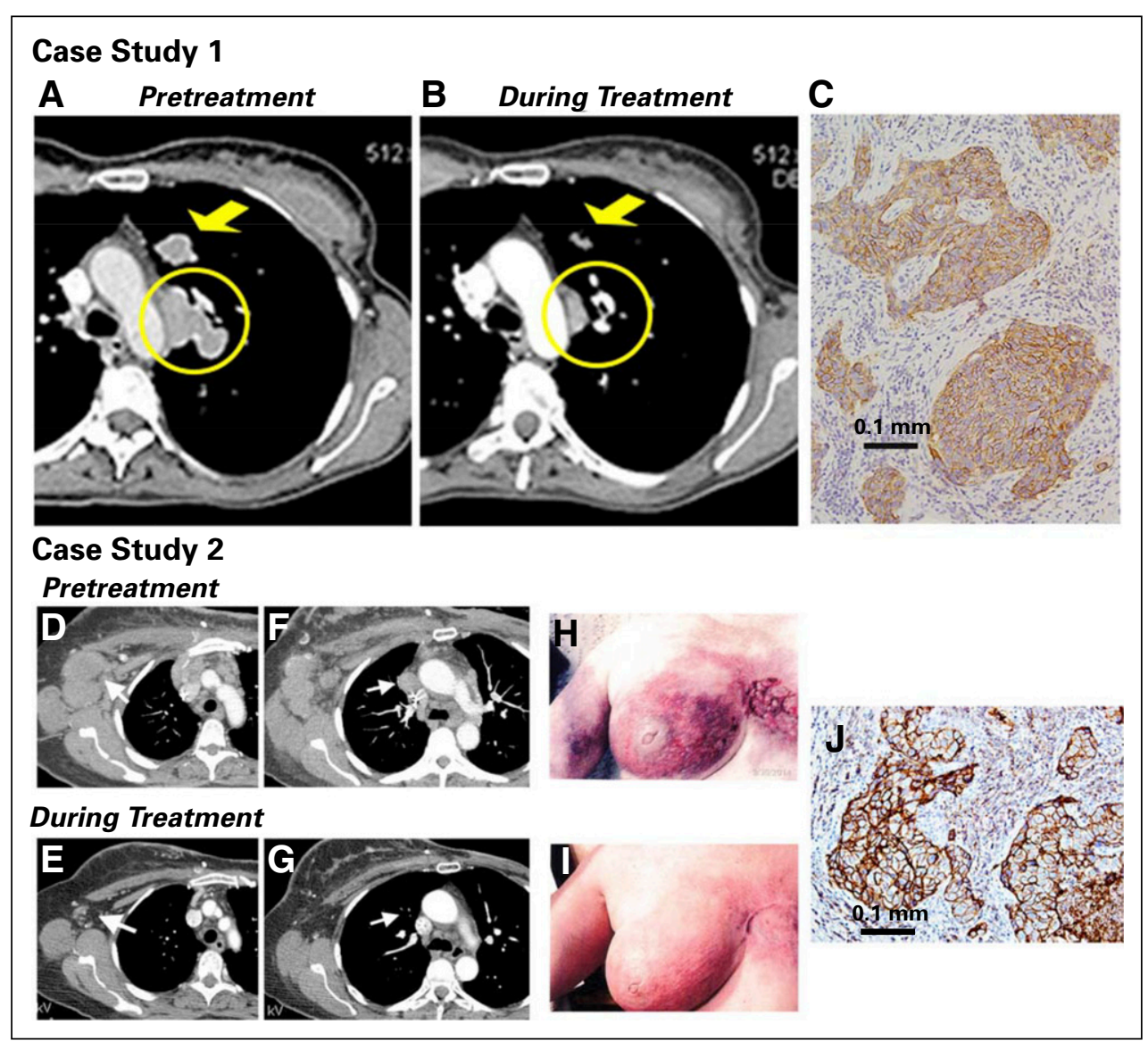

Fig 2. Examples of patients with objective responses. Case study 1: A 48-year-old woman with an initial diagnosis of triple-negative breast cancer in November 2007 received four prior lines of treatment (two lines in a metastatic setting, including an anti-PD-L1 immune checkpoint inhibitor) and presented with lung and lymph node metastases at the time of enrollment in February 2015. She achieved a partial response that started 1.7 months after initiation of treatment with sacituzumab govitecan, with a best response of $54 \%$ reduction at 9.0 months and progression occurring at 14.4 months (partial response duration, 12.7 months). (A) Baseline image of two of the three target lesions: a $24 \times 19$-mm left-upper-lung mass (arrow) and a mediastinal lymph node $(17 \times 29-\mathrm{mm}$; circle). (B) After 16 doses (July 2015$)$, these two target lesions decreased to $13 \times 7$ and $9 \times 19 \mathrm{~mm}$, respectively. (C) Trop-2 expression in an archived tumor specimen by immunohistochemistry that shows $1+$ to $2+$ staining (overall, $2+$ ). Case study 2: A 67-year-old woman with an initial diagnosis of estrogen receptor-positive breast cancer in 2007 that was treated by lumpectomy and local radiation. In 2012, a local recurrence was treated with neoadjuvant doxorubicin and cyclophosphamide followed by taxol and a mastectomy. One year later, the patient received a diagnosis of metastatic disease, which was biopsy-proven triple-negative breast cancer. She was enrolled in a clinical trial and had stable disease for 3 months, but she discontinued treatment because of progression, including new brain metastases. After stereotactic radiosurgery and three cycles of eribulin, the patient progressed and was enrolled in the current study. Her brain metastases had been stable for $>3$ months at the time of enrollment. (D) and (F) Baseline (September 2014) and (E) and (G) 7.2-month follow-up (April 2015) computed tomography scans performed after 22 treatments that showed two target lesions: (D) and (E) axillary and (F) and (G) mediastinal lymph nodes (arrows). Her first computed tomography response assessment in November 2014 showed a $41 \%$ reduction (partial response) after just six doses, which was confirmed 1 month later, and at this time, magnetic resonance imaging showed no evidence of intracranial metastases. (H) Photograph of the patient's skin involvement at the onset of treatment (September 2014) and (I) a photograph that shows a response in December 2014. The patient continued treatment, with 66\% reduction as the overall best response recorded in September 2015 and with four of the five nontarget lesions remaining stable and one completely resolved (supraclavicular lymph node). However, in October 2015, two of the nontarget lesions progressed, and she was withdrawn from the study. (J) Trop-2 expression in archived tumor by immunohistology that shows $3+$ staining.

acceptable safety profile for sacituzumab govitecan, both in animal models ${ }^{17}$ and in the current clinical trial, suggest that in normal tissues, Trop-2 either is poorly accessible (eg, intracellular, restricted to epithelial cells on the luminal side of ducts and glands) or is expressed in lower quantities. In addition, normal tissues that express Trop-2 may not be as sensitive to the cytotoxic effects of SN-38. Therefore, Trop- 2 is an attractive target for the development of novel therapeutics for a variety of epithelial cancers, including TNBC. ${ }^{16-18,22,39,40}$

We determined that almost all $(88 \%)$ of the archival tumor samples in paraffin from these patients expressed relatively high levels of Trop-2 by IHC, which suggests that pretherapy biomarker assessment for patient selection is not required. However, we appreciate that evaluation of Trop- 2 in more-recent biopsy specimens of metastases and/or Trop-2 evaluation in circulating tumor cells may provide important predictive information. Although preliminary, the intensity of staining suggests predictability for improved PFS. However, given the absence of a control group, one cannot claim predictive use of Trop-2 because it could also be a prognostic biomarker. Furthermore, even if the drug is more active in TNBC than in other phenotypes, whether this reflects general greater sensitivity of TNBC to chemotherapy versus specific interaction with Trop-2 expression needs to be determined. Indeed, the Trop-2 staining results should be considered preliminary and hypothesis generating and requires further validation.

This ADC proved to be more effective at a dose equivalent to irinotecan in nonclinical studies because sacituzumab govitecan 


\begin{tabular}{|c|c|c|c|c|}
\hline \multirow[b]{2}{*}{ Response } & \multicolumn{4}{|c|}{ IHC Score $(n=48)$} \\
\hline & $3+$ & $2+$ & $1+$ & 0 \\
\hline \multicolumn{5}{|l|}{ Best overall response } \\
\hline Complete response & 1 & 1 & 0 & 0 \\
\hline Partial response & 7 & 7 & 0 & 0 \\
\hline Stable disease & 14 & 3 & 3 & 1 \\
\hline Progressive disease & 4 & 5 & $1 *$ & $1^{*}$ \\
\hline Median PFS, months & 7.6 & 7.1 & $3.1 \dagger$ & $5.5 \ddagger$ \\
\hline $95 \% \mathrm{Cl}$ & 4.8 to 12.7 & 2.0 to 13.1 & 0.3 to $7.3+$ & 3.6 to $7.1 \neq$ \\
\hline No. of patients & 26 & 16 & $6+$ & $21 \neq$ \\
\hline \multicolumn{5}{|c|}{$\begin{array}{l}\text { Abbreviations: IHC, immunohistochemistry; PFS, progression-free survival. } \\
\text { *These two patients withdrew after one and two doses of sacituzumab govitecan (IMMU-132) without having a computed tomography response assessment but are } \\
\text { considered progressive disease for this analysis. } \\
\text { †Combined IHC scores } 1 \text { and } 0 \text {. } \\
\neq I H C \text { score not available. }\end{array}$} \\
\hline
\end{tabular}

contains seven to eight SN-38 molecules per IgG and delivers more SN-38 to tumors than does irinotecan. ${ }^{16-18,30}$ In addition, sacituzumab govitecan has demonstrated antibody-mediated cellular cytotoxicity in vitro, ${ }^{16}$ which suggests a possible immunotherapeutic function of the ADC that could contribute to the durability of the responses observed in this study.

The patients tolerated prolonged therapy with up to 67 doses given over 23 months, and no evidence of a host immune response to the ADC was present. The treatment had no life-threatening or fatal events, and the toxicity profile was generally mild and manageable, with the primary dose-limiting toxicity from sacituzumab govitecan being neutropenia. Of note, few patients had febrile neutropenia $(7 \%)$ or severe diarrhea (13\%). Thus, the toxicities of this ADC appear to be related primarily to the drug $\mathrm{SN}-38$, but overall incidence and severity are much lower than observed with irinotecan. ${ }^{29}$ The reduced diarrhea and other intestinal toxicity observed with this therapy may be explained in part by sacituzumab govitecan releasing a very low amount of the glucuronidated derivative of $\mathrm{SN}-38$ that is responsible for diarrhea. ${ }^{30}$

In conclusion, single-agent treatment with sacituzumab govitecan was well tolerated and highly active in heavily pretreated patients with mTNBC. These results support further testing of this ADC in TNBC and the potential role of Trop-2 as a predictive biomarker. The study highlights how ADCs against a suitable target and with an appropriate linker could improve the therapeutic index, potentially advancing the selectivity and potency of such cancer therapeutics. On the basis of discussions with regulatory authorities and the Breakthrough Therapy designation for mTNBC from the Food and Drug Administration, the study of a more restricted patient population with at least two prior therapies for metastatic disease is currently underway (NCT01631552).

\section{AUTHORS' DISCLOSURES OF POTENTIAL CONFLICTS OF INTEREST}

Disclosures provided by the authors are available with this article at jco.org.

\section{AUTHOR CONTRIBUTIONS}

Conception and design: Aditya Bardia, David M. Goldenberg, Linda T. Vahdat

Financial support: David M. Goldenberg

Administrative support: David M. Goldenberg

Provision of study materials or patients: Aditya Bardia, Ingrid A. Mayer, Jennifer R. Diamond, Rebecca L. Moroose, Steven J. Isakoff, Alexander N. Starodub, Nikita C. Shah, Serengulam V. Govindan, Linda T. Vahdat Collection and assembly of data: Aditya Bardia, Ingrid A. Mayer, Jennifer R. Diamond, Rebecca L. Moroose, Steven J. Isakoff, Alexander N. Starodub, Nikita C. Shah, Joyce O'Shaughnessy, Kevin Kalinsky, Michael Guarino, Vandana Abramson, Dejan Juric, Sara M. Tolaney, Jordan Berlin, Wells A. Messersmith, Allyson J. Ocean, William A. Wegener, Pius Maliakal,

Robert M. Sharkey, Linda T. Vahdat

Data analysis and interpretation: Aditya Bardia, Ingrid A. Mayer,

Jennifer R. Diamond, Rebecca L. Moroose, Steven J. Isakoff, Robert M. Sharkey, Serengulam V. Govindan, David M. Goldenberg, Linda T. Vahdat Manuscript writing: All authors

Final approval of manuscript: All authors

Accountable for all aspects of the work: All authors

\section{REFERENCES}

1. Sledge GW, Mamounas EP, Hortobagyi GN, et al: Past, present, and future challenges in breast cancer treatment. J Clin Oncol 32:1979-1986, 2014

2. Torre LA, Bray $F$, Siegel RL, et al: Global cancer statistics, 2012. CA Cancer J Clin 65:87-108, 2015

3. Perou CM, Sørlie T, Eisen MB, et al: Molecular portraits of human breast tumours. Nature 406: 747-752, 2000
4. Carey LA, Perou CM, Livasy CA, et al: Race, breast cancer subtypes, and survival in the Carolina Breast Cancer Study. JAMA 295:2492-2502, 2006

5. Hurvitz S, Mead M: Triple-negative breast cancer: Advancements in characterization and treatment approach. Curr Opin Obstet Gynecol 28: 59-69, 2016

6. Keenan T, Moy B, Mroz EA, et al: Comparison of the genomic landscape between primary breast cancer in African American versus white women and the association of racial differences with tumor recurrence. J Clin Oncol 33:3621-3627, 2015
7. Rugo HS, Olopade Ol, DeMichele A, et al: Adaptive randomization of veliparib-carboplatin treatment in breast cancer. N Engl J Med 375:23-34, 2016

8. André $F$, Zielinski CC: Optimal strategies for the treatment of metastatic triple-negative breast cancer with currently approved agents. Ann Oncol 23:vi46-vi51, 2012 (suppl 6)

9. Dent R, Trudeau M, Pritchard KI, et al: Triplenegative breast cancer: Clinical features and patterns of recurrence. Clin Cancer Res 13:4429-4434, 2007

10. Forero-Torres $A$, Varley $K E$, Abramson VG, et al: TBCRC 019: A phase II trial of nanoparticle 
albumin-bound paclitaxel with or without the antideath receptor 5 monoclonal antibody tigatuzumab in patients with triple-negative breast cancer. Clin Cancer Res 21:2722-2729, 2015

11. Isakoff SJ, Mayer EL, He L, et al: TBCRC009: A multicenter phase II clinical trial of platinum monotherapy with biomarker assessment in metastatic triplenegative breast cancer. J Clin Oncol 33:1902-1909, 2015

12. Lin NU, Vanderplas $A$, Hughes $M E$, et al: Clinicopathologic features, patterns of recurrence, and survival among women with triple-negative breast cancer in the National Comprehensive Cancer Network. Cancer 118:5463-5472, 2012

13. O'Shaughnessy J, Schwartzberg L, Danso MA, et al: Phase III study of iniparib plus gemcitabine and carboplatin versus gemcitabine and carboplatin in patients with metastatic triple-negative breast cancer. J Clin Oncol 32:3840-3847, 2014

14. Perez EA, Patel T, Moreno-Aspitia A: Efficacy of ixabepilone in ER/PR/HER2-negative (triple-negative) breast cancer. Breast Cancer Res Treat 121:261-271, 2010

15. Cardoso F, Costa A, Norton L, et al: ESO-ESMO 2nd International Consensus Guidelines for Advanced Breast Cancer (ABC2). Breast 23:489-502, 2014

16. Cardillo TM, Govindan SV, Sharkey RM, et al: Sacituzumab govitecan (IMMU-132), an anti-Trop-2/ SN-38 antibody-drug conjugate: Characterization and efficacy in pancreatic, gastric, and other cancers. Bioconjug Chem 26:919-931, 2015

17. Cardillo TM, Govindan SV, Sharkey RM, et al: Humanized anti-Trop-2 lgG-SN-38 conjugate for effective treatment of diverse epithelial cancers: Preclinical studies in human cancer xenograft models and monkeys. Clin Cancer Res 17:3157-3169, 2011

18. Goldenberg DM, Cardillo TM, Govindan SV, et al: Trop-2 is a novel target for solid cancer therapy with sacituzumab govitecan (IMMU-132), an antibodydrug conjugate (ADC). Oncotarget 6:22496-22512, 2015

19. Shvartsur A, Bonavida B: Trop2 and its overexpression in cancers: Regulation and clinical/therapeutic implications. Genes Cancer 6:84-105, 2015

20. Ambrogi $F$, Fornili $M$, Boracchi $P$, et al: Trop-2 is a determinant of breast cancer survival. PLoS One 9:e96993, 2014 [Erratum: PLoS One 9:e110606, 2014]
21. Lipinski M, Parks DR, Rouse RV, et al: Human trophoblast cell-surface antigens defined by monoclonal antibodies. Proc Natl Acad Sci U S A 78:5147-5150, 1981

22. Stepan LP, Trueblood ES, Hale K, et al: Expression of Trop2 cell surface glycoprotein in normal and tumor tissues: Potential implications as a cance therapeutic target. J Histochem Cytochem 59: 701-710, 2011

23. Basu A, Goldenberg DM, Stein R: The epithelial/carcinoma antigen EGP-1, recognized by monoclonal antibody RS7-3G11, is phosphorylated on serine 303. Int J Cancer 62:472-479, 1995

24. Wang J, Day R, Dong $Y$, et al: Identification of Trop-2 as an oncogene and an attractive therapeutic target in colon cancers. Mol Cancer Ther 7:280-285, 2008

25. Lin H, Huang JF, Qiu JR, et al: Significantly upregulated TACSTD2 and cyclin D1 correlate with poor prognosis of invasive ductal breast cancer. Exp Mol Pathol 94:73-78, 2013

26. Rothenberg ML: Topoisomerase I inhibitors: Review and update. Ann Oncol 8:837-855, 1997

27. Hayashi $H$, Tsurutani J, Satoh $T$, et al: Phase II study of bi-weekly irinotecan for patients with previously treated HER2-negative metastatic breast cancer: KMBOG0610B. Breast Cancer 20:131-136, 2013

28. Perez EA, Hillman DW, Mailliard JA, et al: Randomized phase II study of two irinotecan schedules for patients with metastatic breast cancer refractory to an anthracycline, a taxane, or both. J Clin Oncol 22:2849-2855, 2004

29. Mathijssen $\mathrm{RH}$, van Alphen RJ, Verweij J, et al: Clinical pharmacokinetics and metabolism of irinotecan (CPT-11). Clin Cancer Res 7:2182-2194, 2001

30. Sharkey RM, McBride WJ, Cardillo TM, et al: Enhanced delivery of SN-38 to human tumor xenografts with an anti-Trop-2-SN-38 antibody conjugate (sacituzumab govitecan). Clin Cancer Res 21: 5131-5138, 2015

31. Starodub AN, Ocean AJ, Shah MA, et al: Firstin-human trial of a novel anti-Trop-2 antibody-SN-38 conjugate, sacituzumab govitecan, for the treatment of diverse metastatic solid tumors. Clin Cancer Res 21:3870-3878, 2015

31a. Hammond $M E H$, Hayes DF, Dowsett $M$, et al: American Society of Clinical Oncology/College of American Pathologists guideline recommendations for immunohistochemical testing of estrogen and progesterone receptors in breast cancer. J Clin Oncol 28:2784-2795, 2010

31b. Wolff AC, Hammond MEH, Hicks DG, et al: Recommendations for human epidermal growth factor receptor 2 testing in breast cancer: American Society of Clinical Oncology/College of American Pathologists clinical practice guideline update. J Clin Oncol 31:3997-4013, 2013

31c. Medical Dictionary for Regulatory Activities version 10, www.meddra.org

32. Govindan SV, Sharkey RM, Goldenberg DM: Prospects and progress of antibody-drug conjugates in solid tumor therapies. Expert Opin Biol Ther 16: 883-893, 2016

33. Sievers EL, Senter PD: Antibody-drug conjugates in cancer therapy. Annu Rev Med 64:15-29, 2013

34. Slamon DJ, Leyland-Jones B, Shak S, et al: Use of chemotherapy plus a monoclonal antibody against HER2 for metastatic breast cancer that overexpresses HER2. N Engl J Med 344:783-792, 2001

35. Verma S, Miles D, Gianni L, et al: Trastuzumab emtansine for HER2-positive advanced breast cancer. N Engl J Med 367:1783-1791, 2012

36. Xie $R$, Mathijssen $R H$, Sparreboom A, et al: Clinical pharmacokinetics of irinotecan and its metabolites in relation with diarrhea. Clin Pharmacol Ther 72:265-275, 2002

37. Stein R, Chen S, Sharkey RM, et al: Murine monoclonal antibodies raised against human non-small cell carcinoma of the lung: Specificity and tumor targeting. Cancer Res 50:1330-1336, 1990

38. Cubas R, Li M, Chen C, et al: Trop2: A possible therapeutic target for late stage epithelia carcinomas. Biochim Biophys Acta 1796:309-314, 2009

39. Stein R, Basu A, Chen S, et al: Specificity and properties of MAb RS7-3G11 and the antigen defined by this pancarcinoma monoclonal antibody. Int $J$ Cancer 55:938-946, 1993

40. Trerotola M, Cantanelli P, Guerra E, et al: Upregulation of Trop-2 quantitatively stimulates human cancer growth. Oncogene 32:222-233, 2013

Aditya Bardia, Steven J. Isakoff, and Dejan Juric, Massachusetts General Hospital Cancer Center; Aditya Bardia, Steven J. Isakoff, Dejan Juric, and Sara M. Tolaney, Harvard Medical School; Sara M. Tolaney, Dana-Farber Cancer Institute, Boston, MA; Ingrid A. Mayer, Vandana Abramson, and Jordan Berlin, Vanderbilt-Ingram Cancer Center, Nashville, TN; Jennifer R. Diamond and Wells A. Messersmith, University of Colorado Cancer Center, Aurora, CO; Rebecca L. Moroose and Nikita C. Shah, University of Florida Health Cancer Center, Orlando, FL; Alexander N. Starodub, Indiana University Health Center for Cancer Care, Goshen, IN; Joyce O’Shaughnessy, Texas Oncology-Baylor Charles A. Sammons Cancer Center; Joyce O’Shaughnessy, US Oncology, Dallas, TX; Kevin Kalinsky, Columbia University Herbert Irving Comprehensive Cancer Center; Allyson J. Ocean and Linda T. Vahdat, Weill Cornell Medicine, New York, NY; Michael Guarino, Helen F. Graham Cancer Center, Newark, DE; William A. Wegener, Pius Maliakal, Robert M. Sharkey, Serengulam V. Govindan, and David M. Goldenberg, Immunomedics, Morris Plains, NJ.

Supported in part by Immunomedics.

\section{Support}

\section{Prior Presentation}

Presented at the AACR-NCI-EORTC International Conference on Molecular Targets and Cancer Therapeutics, Boston, MA, November 5-9, 2015; 38th Annual San Antonio Breast Cancer Symposium, San Antonio, TX, December 8-12, 2015; 12th Annual Protein and Antibody Engineering Summit, Boston, MA, April 25-29, 2016; and 39th Annual San Antonio Breast Cancer Symposium, San Antonio, TX, December 6-10, 2016. 


\section{AUTHORS' DISCLOSURES OF POTENTIAL CONFLICTS OF INTEREST}

Efficacy and Safety of Anti-Trop-2 Antibody Drug Conjugate Sacituzumab Govitecan (IMMU-132) in Heavily Pretreated Patients With Metastatic Triple-Negative Breast Cancer

The following represents disclosure information provided by authors of this manuscript. All relationships are considered compensated. Relationships are self-held unless noted. I = Immediate Family Member, Inst = My Institution. Relationships may not relate to the subject matter of this manuscript. For more information about ASCO's conflict of interest policy, please refer to www.asco.org/rwc or ascopubs.org/jco/site/ifc.

Aditya Bardia

Consulting or Advisory Role: Novartis, Genentech

Ingrid A. Mayer

Consulting or Advisory Role: Novartis

Research Funding: Novartis, Clovis Oncology, Pfizer

\section{Jennifer R. Diamond}

Research Funding: CASI Pharmaceuticals, Millennium Pharmaceuticals (Inst), Genentech (Inst), Roche (Inst), OncoMed Pharmaceuticals (Inst), Bristol-Myers Squibb (Inst), Bayer AG (Inst), MedImmune (Inst), Taiho Pharmaceutical (Inst), SCRI Development Innovations (Inst)

Travel, Accommodations, Expenses: Takeda Pharmaceuticals

\section{Rebecca L. Moroose}

Honoraria: Genentech, Pfizer, Biotheranostics

Consulting or Advisory Role: Pfizer, Biotheranostics

Speakers' Bureau: Pfizer, Genentech

\section{Steven J. Isakoff}

Consulting or Advisory Role: Myriad Genetics, Merrimack

Pharmaceuticals, AbbVie, PharmaMar

Research Funding: Genentech, PharmaMar, AbbVie, OncoPep

Travel, Accommodations, Expenses: Myriad Genetics

Alexander N. Starodub

Consulting or Advisory Role: Sandoz, Bayer AG

Speakers' Bureau: Bristol-Myers Squibb

Travel, Accommodations, Expenses: Bayer AG, Bristol-Myers Squibb, Sandoz

\section{Nikita C. Shah}

Honoraria: Novartis, Eisai, Pfizer

Consulting or Advisory Role: Eisai, Pfizer

Speakers' Bureau: Eisai, Pfizer, Novartis

\section{Joyce O'Shaughnessy}

Honoraria: AstraZeneca, Eli Lilly, AbbVie, Arno Therapeutics, Celgene, Nektar, Eisai, Novartis, Pfizer, Genentech, Roche

Consulting or Advisory Role: Novartis, Pfizer, Eli Lilly, Arno Therapeutics, AbbVie, AstraZeneca, Celgene, Nektar, Genentech, Roche, Eisai

Travel, Accommodations, Expenses: Celgene, Nektar, Eli Lilly, Novartis, Pfizer, AbbVie

\section{Kevin Kalinsky}

Stock or Other Ownership: Novartis (I)

Consulting or Advisory Role: Biotheranostics, Eli Lilly, Pfizer, Amgen, Novartis, Eisai

Michael Guarino

No relationship to disclose

\section{Vandana Abramson}

Consulting or Advisory Role: Novartis

Research Funding: Genentech, Roche, Astellas Pharma

\section{Dejan Juric}

Consulting or Advisory Role: Novartis, Eisai, EMD Serono

Research Funding: Novartis, Genentech

\section{Sara M. Tolaney}

Consulting or Advisory Role: Pfizer, Nektar, AstraZeneca

Research Funding: Genentech, Pfizer, Merck, Exelixis, Novartis

\section{Jordan Berlin}

Consulting or Advisory Role: Celgene, Symphogen, Genentech, Roche, Vertex Pharmaceuticals, Eli Lilly, ImClone Systems, Novartis, EMD Serono, Ipsen, Boston Biomedical, Pharmacyclics, Threshold Pharmaceuticals, Aduro Biotech, Cornerstone Pharmaceuticals, ARMO Biosciences, Five Prime Therapeutics, Opsona Therapeutics Research Funding: Genentech (Inst), Roche (Inst), OncoMed (Inst), Novartis (Inst), Immunomedics (Inst), AbbVie (Inst), Gilead Sciences (Inst), Merrimack Pharmaceuticals (Inst), Taiho Pharmaceutical (Inst), Five Prime Therapeutics (Inst), Loxo Oncology(Inst), Vertex

Pharmaceuticals (Inst), Bayer AG (Inst), Symphogen (Inst), Incyte (Inst), Pharmacyclics (Inst)

Travel, Accommodations, Expenses: AbbVie, Genentech, Roche, Janssen Pharmaceuticals, Celgene, Boston Biomedical

Other Relationship: Momenta Pharmaceuticals, Symphogen, AstraZeneca

Wells A. Messersmith

Consulting or Advisory Role: OncoMed, Immunomedics, Gilead Sciences Research Funding: Pfizer, Roche, Genentech, Millennium Pharmaceuticals, OncoMed, Immunomedics

Allyson J. Ocean

Speakers' Bureau: Genentech, Ipsen, Merrimack Pharmaceuticals

William A. Wegener

Employment: Immunomedics

Stock or Other Ownership: Immunomedics

Pius Maliakal

Employment: Immunomedics

Stock or Other Ownership: Immunomedics

Robert M. Sharkey

Employment: Immunomedics

Stock or Other Ownership: Immunomedics

Honoraria: Immunomedics

Serengulam V. Govindan

Employment: Immunomedics

Stock or Other Ownership: Immunomedics

Patents, Royalties, Other Intellectual Property: Coinventorship in patents and patent applications assigned to Immunomedics

\section{David M. Goldenberg}

Employment: Immunomedics, Immunomedics (I)

Leadership: Immunomedics, Immunomedics (I)

Stock or Other Ownership: Immunomedics, Immunomedics (I)

Patents, Royalties, Other Intellectual Property: Patent protection for multiple antibody-based products and procedures

Travel, Accommodations, Expenses: Immunomedics, Immunomedics (I)

Linda T. Vahdat

Consulting or Advisory Role: BERG

Speakers' Bureau: Eisai

Research Funding: Celldex Therapeutics (Inst), Polyphor (Inst), Clovis Oncology (Inst), Immunomedics (Inst), AstraZeneca (Inst), PharmaMar (Inst), AbbVie (Inst), Galena Biopharma (Inst), Optimer Biotechnology (Inst), Millennium Pharmaceuticals (Inst)

Patents, Royalties, Other Intellectual Property: Patent pending on the application for BMD progenitor cells (Inst) 


\section{Appendix}

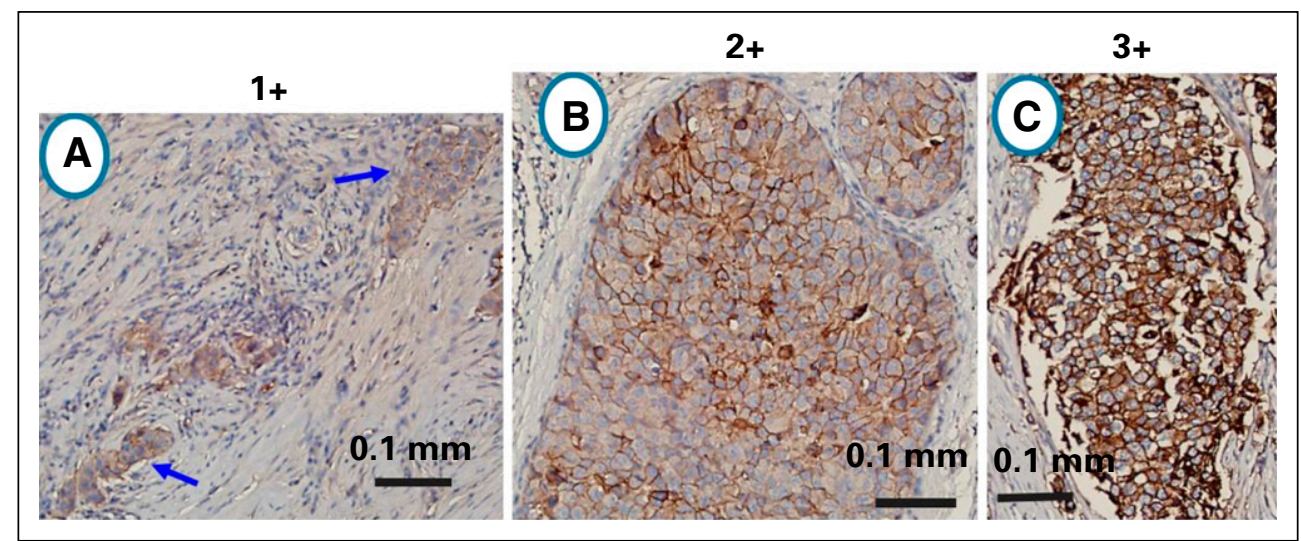

Fig A1. Example of scoring for Trop-2 staining. (A) 1+, generally weak membrane or cytoplasmic staining in $>10 \%$ of tumor cells; (B) $2+$, moderately stained membrane or cytoplasm in $>10 \%$ of tumor cells; (C) $3+$, strongly stained membrane or cytoplasm in $>10 \%$ of tumor cells.

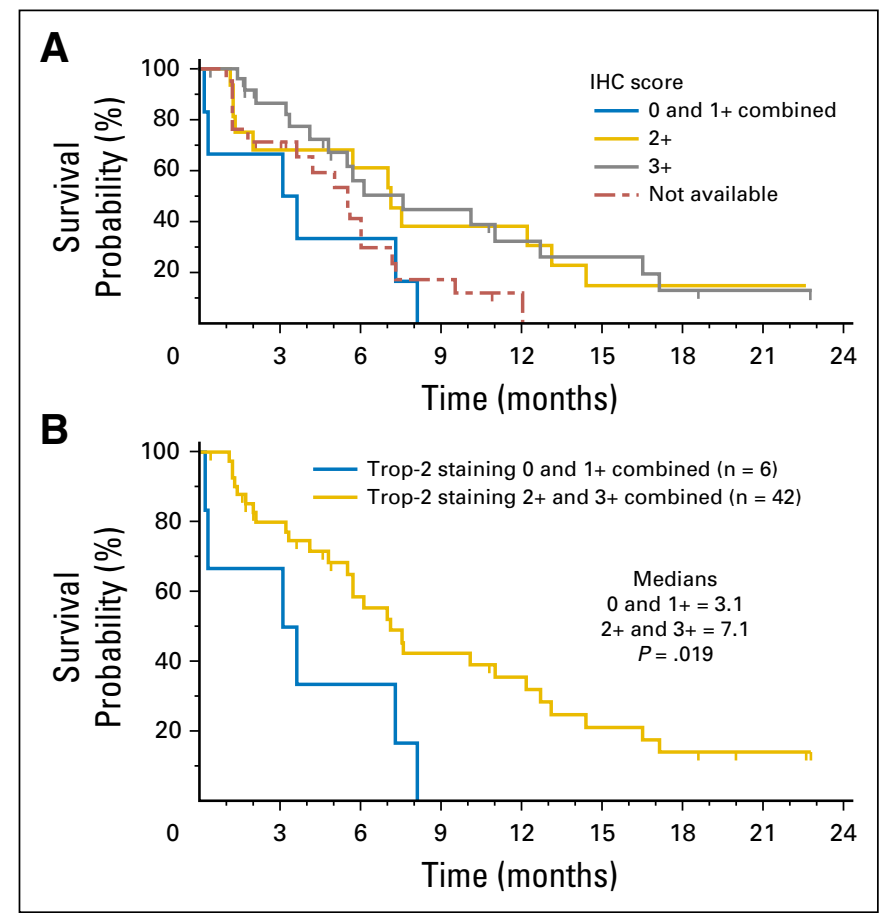

Fig A2. Trop-2 IHC scoring vs. PFS $(n=48)$. (A) Kaplan-Meier plot for 4 separate groups with the $0(n=2)$ and $1+(n=4)$ combined, including 21 patients who did not have a specimen for Trop-2 analysis. (B) Kaplan-Meier plot comparing PFS for patients with specimens stained 0 and $1+$ combined vs. those with $2+$ and $3+$ Trop-2 staining combined. 